\title{
An outbreak of Streptococcus equi subsp. zooepidemicus in layers: evidence of fecal transmission.
}

\begin{abstract}
Now and then, major disease outbreaks caused by Streptococcus equi subsp. zooepidemicus are reported in poultry. Besides acute septicemia, infection can result in a peracute or chronic form of disease with described mortality rates of $11 \%$ to $80 \%$. Previously, source of infection in poultry was linked to horses in which this bacteria can be present as an opportunistic pathogen on mucus membranes. The main route of spreading and maintaining within a poultry flock, after entering the stable, however, remains unclear. This case report describes an outbreak of Streptococcus equi subsp. zooepidemicus affecting a flock of 28,500 layer hens housed in an aviary system with free-range. Efforts to reveal the route of dissemination of the Streptococcus bacteria within the flock assume fecal excretion by clinically healthy chickens.
\end{abstract}

Keywords: Streptococcus equi subsp. zooepidemicus; outbreak; layers; free range; fecal transmission; carrier

\section{Introduction}

Streptococcus equi subsp. zooepidemicus (S. equi subsp. zooepidemicus) is an opportunistic pathogen found on mucus membranes of healthy horses, in which it is associated with mainly uterine and respiratory tract infections as well as ulcerative keratitis. The pathogen can also cause disease in humans and a wide range of animals (Webb et al., 2008) including adult poultry. In poultry, infection with S. equi subsp. zooepidemicus often results in acute septicemia characterized by sudden death. Additionally, a peracute and a chronic form of infection are described in which affected birds are showing signs of lethargy, emaciation, respiratory symptoms, diarrhea and cyanosis (Edwards \& Hull, 1937; Buxton, 1952, Sato et al., 1960, Peckham, 1966). In breeders and layers, egg production drops of as much as $50 \%$ have been described (Bisgaard et al., 2012). Mortality in an affected flock can range from $11 \%$ to $80 \%$ (Genest \& Nadau, 1944, Buxton, 1952, Sato \& Miura, 1960, Bisgaard et al., 2012). Related to the strict biosecurity measures implemented in industrial poultry production, S. equi subsp. zooepidemicus outbreaks in poultry are relatively uncommon (Webb et al., 2008; Thayer \& Waltman, 2013). Yet, it has been hypothesized that the present increase in outdoor production might result in a higher appearance of these infections in poultry (Bisgaard et al., 2012). Previously, the source of entrance of this opportunistic pathogen into the poultry farm has been linked to horses (Bisgaard et al., 2012). The main route of spreading and maintaining of the bacteria within the flock is not yet elucidated. In the present paper, a recent outbreak of $S$. equi subsp. zooepidemicus affecting layers housed in an aviary system with free range is reported. In addition, efforts were made to reveal the route of dissemination of the Streptococcus infection within the affected flock.

\section{Materials and methods}

Flock history. In the spring of 2018, high mortality was observed in a flock of 28,500 ISA Brown layers with untreated beaks and kept in aviary housing system with free range. Besides layers, calves and two horses were present on the farm. From 44 weeks of age onwards, weekly mortality rates in the layer flock gradually raised from $1.1 \%$ till $2.9 \%$ at 50 weeks of age. The chickens were found dead without prior symptoms. Feed intake and egg production in surviving birds (90\%) were steady. Dead birds showed signs of cloacal pecking and cannibalism. On-farm necropsy, performed by the company veterinarian, revealed signs of sepsis including splenomegaly, enlarged hearts and pinpoint bleedings in the muscles. After a treatment with phenoxymethylpenicillin (Dechra Veterinary Products NV., Belgium) dosed at $20 \mathrm{mg} / \mathrm{kg}$ body weight/day for 5 consecutive days in the drinking water, a periodical decline in mortality was observed. After a fortnight, mortality increased again (weekly mortality rate of $3.7 \%)$. Besides sudden deaths, clinical signs of depression were noticed. Ten layers with signs of somnolence were euthanized and from each individual laying hen a pooled sample from liver and spleen $(n=10)$ was collected by the company veterinarian for bacteriologic culture on commercial growth media. E. coli and $S$. equi subsp. zooepidemicus were isolated from 8 and 3 pooled spleen/liver samples, respectively. Based on these results, the phenoxymethylpenicillin treatment was repeated using a higher dose of $25 \mathrm{mg} / \mathrm{kg}$ bodyweight. Egg production dropped from $90 \%$ to $86 \%$, but mortality rates improved. Still, when 54 weeks of age, 350 layers were found dead in the stable, once more. At that time point, it was decided to send chickens to the Department of Pathology, Bacteriology and Avian Diseases, Faculty of Veterinary Medicine, Ghent University for postmortem examination.

Necropsy. Ten birds found dead in the stable and 10 clinically ill birds showing signs of depression, were delivered for postmortem examination. Necropsy procedures were as follows: living birds were euthanized by means of 
cervical dislocation. After external inspection, birds were weighed, carcasses were plucked, skinned and sternum removed. A macroscopic investigation of all tissues and internal organs was performed. Next, affected organs were collected for microbiological and histopathological examination as described below. Six weeks later, an additional necropsy was performed on 10 clinically healthy birds to investigate the route of S. equi subsp. zooepidemicus excretion in the flock. From these birds, cloacal swabs using cotton-tipped aluminium shafted swabs (Copan Diagnostics Inc., Corona, USA) and cecal contents were collected.

Histopathology. Samples of affected hearts $(n=2)$, livers $(n=2)$, spleens $(n=2)$ were fixed for $24 \mathrm{~h}$ in $10 \%$ buffered formalin and embedded in paraffin. Sections of the tissue were stained with hematoxylin and eosin and microscopically examined.

Bacteriology. Bacterial cultures of affected organs, originating from 11 layers, were made on commercial growth media, including Columbia agar plates with $5 \%$ sheep blood and McConkey III agar (Oxoid, Hampshire, UK). Hens with signs of cloacal pecking or cannibalism $(n=6)$ or hens without macroscopic organ lesions $(n=3)$ were excluded from bacteriological analysis. Growth media were inspected for bacterial growth after $24 \mathrm{~h}$ of incubation at $35^{\circ} \mathrm{C}$ in a $5 \% \mathrm{CO}_{2}$ enriched atmosphere. Cloacal swabs, collected from 10 healthy chickens, were cultured on Columbia CNA agar plates with $5 \%$ sheep blood agar (CNA; Oxoid, Hampshire, UK) for selective isolation of Gram positive bacteria. The cecal contents collected from the healthy chickens were diluted 1:9 (weight/volume) in Phosphate buffered Saline and tenfold serial dilutions were inoculated onto CNA agar plates. After $24 \mathrm{~h}$ of incubation in a $5 \% \mathrm{CO}_{2}$ enriched atmosphere at $35^{\circ} \mathrm{C}$, plates were checked for presence of colonies with $\mathrm{S}$. equi subsp. zooepidemicus characteristics (Roy et al., 2013). For the cecal contents, viable counts were made and the number of colony forming units (cfu)/g cecal content was calculated. To investigate the link with the on-farm horses, swabs from the nasal and vaginal mucosa were collected and pre-enriched in Columbia broth (Oxoid, Hampshire, UK) supplemented with colistin $(10 \mu \mathrm{g} / \mathrm{ml})$ and nalidixic acid $(5 \mu \mathrm{g} / \mathrm{ml})$. Subsequently, samples were incubated overnight at $37^{\circ} \mathrm{C}$ and a loopful of the suspensions was plated on CNA agar plates and incubated in a 5\% CO2 enriched atmosphere for $24 \mathrm{~h}$ at $37^{\circ} \mathrm{C}$.

After culture, bacterial colonies were identified with Matrix Assisted Laser Desorption/lonisation Time-Of-Flight Mass Spectrometry (MALDI-TOF MS) using the direct transfer method and $\alpha$-cyano-4-hydroxycinnamic acid (HCCA) as a matrix, according to the manufacturer's guidelines. The spectra were obtained and analysed with the MBT Compass software version 4.1 (Bruker Daltonik), and compared with a database of 6120 mean spectra projections. Identifications with a score value $>2.000$ were taken into account as reliable to the species level.

Because of the treatment failure, the antimicrobial susceptibility of the $S$. equi subsp. zooepidemicus isolates against penicillines was determined using the disc diffusion test according to the guidelines of the Clinical and Laboratory Standards Institute (CLSI, 2008).

\section{Results}

Necropsy. Postmortem examinations on the dead birds $(n=10)$ showed 4 hens without and 6 hens with signs of cloacal pecking and/or cannibalism. Septicemia was observed in all $(n=10)$ birds including spleno- and hepatomegaly $(n=5)$, renomegaly $(n=1)$ and enlarged hearts showing signs of necrotic myocarditis $(n=2)$ (Figure 1). Also more productive lesions such as fibrinous perihepatitis $(n=1)$, fibrinous perifolliculitis $(n=4)$, fibrinous coelomitis $(n=3)$, fibrinous endocarditis $(n=1)$, fibrinous pericarditis $(n=2)$ and chronic salpingitis $(n=1)$ were observed.

In the clinically ill birds, euthanized for necropsy $(n=10)$, similar lesions were observed in 7 birds: spleno- en hepatomegaly $(n=3)$, necrotic myocarditis $(n=4)$, fibrinous pericarditis $(n=4)$, perihepatitis $(n=4)$, perifolliculitis $(n=4)$ and coelomitis $(n=4)$. In 3 birds significant macroscopic lesions were not present.

In the clinically healthy birds, euthanized for necropsy $(n=10)$ organ lesions were not observed and normal ovary activity was present.

Histopathology. Histopathological examination of two hearts both showed multifocal to diffuse necrosis of the subepicardial cardiomyocytes with disruption and disarray of the muscle bundles, loss of nuclei, loss of muscle striation and accumulation of cellular debris. Within the debris, large numbers of heterophiles, plasma cells, lymphocytes and macrophages, as well as large amounts of eosinophilic fibrillar material (fibrinous epicarditis) were present combined with a minimal amount of collagen. There were multifocal colonies of coccoid bacteria. Capillaries and small arteries close to the epicardium are diffusely enlarged and engorged with erythrocytes (congestion). Some vessels showed mild disruption of the vascular wall with moderate perivascular accumulation 
of macrophages, plasma cells and lymphocytes (vasculitis). One small artery contained a fibrin thrombus within the vascular lumen (thrombosis).

In one liver there were multiple colonies of coccoid bacteria in the sinusoids as well as in some small hepatic vessels with degeneration of the surrounding hepatocytes. Larger blood vessels were distended by an accumulation of eosinophilic fibrillar material (fibrin thrombus). The portal tracts in the other liver were multifocally infiltrated with moderate numbers of lymphocytes and plasma cells.

Within one spleen there was marked deposition of pale homogeneous eosinophilic material, cellular debris and some nuclear fragments centrally within the follicles (necrosis) surrounded by large numbers of macrophages, as well as lower numbers of lymphocytes and plasma cells. The other spleen showed lower number and smaller sizes of lymphoid follicles (atrophy) with multifocal aggregates of hemosiderin-laden macrophages. The splenic capsule of both spleens was covered by a layer of fibrin with enmeshed lymphocytes, plasma cells and macrophages (fibrinous serositis).

Microbiology. Results of the bacteriological analysis of the hens are shown in Table 1.

From the 11 hens of which affected organs were collected for bacteriology, 6 proved positive for S. equi subsp. zooepidemicus. In 4 hens, co-infection with $E$. coli was observed. Three hens were positive for $E$. coli, only. The S. equi subsp. zooepidemicus isolates were susceptible for penicillines, as determined by disc diffusion test.

We were unable to culture S. equi subsp. zooepidemicus out of the cloacal swabs, collected from healthy hens. Yet, substantial numbers of $S$. equi subsp. zooepidemicus (i.e. $4 \times 10^{2} \mathrm{cfu} / \mathrm{g}$ ) were counted in the ceca of one of these hens after bacteriologic titration.

In addition, we were unable to culture S. equi subsp. zooepidemicus from the nasal samples collected from the horses.

\section{Discussion}

This case report describes an outbreak of S. equi subsp. zooepidemicus in layers housed in an aviary system with free-range. Clinical signs and postmortem findings observed during the present outbreak are in accordance with previous reports (Edwards \& Hull, 1937; Buxton, 1952; Sato \& Miura, 1960; Peckham, 1966; Bisgaard et al, 2012). Previously, the source of S. equi subsp. zooepidemicus infection has been linked to horses (Bisgaard et al., 2012). Also in the current outbreak, two horses were present on the farm. Mucosal samples of the noses and vaginas were examined, but $S$. equi subsp. zooepidemicus could not be isolated. However, using bacterial culture, false negative results might not be excluded.

Healthy carriers of S. equi subsp. zooepidemicus have been encountered in mammals. In poultry, however, this was not yet demonstrated. Bisgaard et al. (2012), checked for the presence of S. equi subsp. zooepidemicus in cloacal swabs taken from healthy chickens in an affected layer flock, but could not isolate the bacteria. Also in the current study, cloacal swabs, originating from 10 apparently healthy chickens, proved negative for S. equi subsp. zooepidemicus using culture. Yet, bacterial titration revealed substantial numbers of $S$. equi subsp. zooepidemicus present in the ceca of a healthy chicken. This result hypothesizes that transmission of the infection within the flock might occur through the fecal route. Presence of subclinical carriers might also explain why the infection can persist or re-occur in a flock.

Cloacal pecking and cannibalism have been observed in S. equi subsp. zooepidemicus outbreaks (present study; Bisgaard et al. 2012). Taking into account that streptococci can be present in feces, this might enhance oral uptake and dissemination of the bacteria within a flock. Also bacteremia through footpad lesions, as suggested by Van Roy et al. (2013) might be a possible infection route, when fecal excretion is assumed.

In conclusion, in this case report an outbreak of $S$. equi subsp. zooepidemicus affecting a flock of 28,500 layer hens housed in an aviary system with free-range is described. Efforts to reveal the route of dissemination of the Streptococcus bacteria within the flock assume fecal excretion by clinically healthy chickens.

\section{Acknowledgements}

The authors would like to thank Arlette Van de Kerckhove and Serge Verbanck for their excellent technical assistance. The MALDI-TOF mass spectrometer was financed by the Research Foundation Flanders (FWOVlaanderen) as Hercules project GOH2516N (AUGE/15/05). 


\section{References}

Bisgaard, M., Bojesen, A.M., Petersen, M.R. \& Christensen, H. (2012). A major outbreak of Streptococcus equi subsp. zooepidemicus infections in free-range chickens is linked to horses. Avian Diseases, 56, 561-566.

Buxton, J.C. (1952). Disease in poultry associated with Streptococcus zooepidemicus. The Veterinary Record, 64, 221-223.

Edwards, P.R. \& Hull, F.E. (1937). Hemolytic streptococci in chronic peritonitis and salpingitis of hens. Journal of the American Veterinary Medical Association, 44, 656-660.

Clinical and Laboratory Standards Institute. (2008). Performance Standards for Antimicrobial Disk and Dilution Susceptibility Tests for Bacteria Isolated From Animals-3rd edn: Approved Standard M31-A3. CLSI, Wayne, PA, USA.

Genest, P. \& Nadeau, J.D. (1944). Observation, chez la poule, d'une epizootie due a Streptococcus zooepidemicus. Canadian Journal of the Comparative Medicine and Veterinary Science, 8, 342-349.

Peckham, M.C. (1966). An outbreak of Streptococcosis (Apoplectiform septicemia) in white rock chickens. Avian Diseases, 10, 413-421.

Roy, K., Bisgaard, M., Kyvsgaard, N.C., Christensen, J.P., Nielsen, O.L., Biswas, P.K., Pors, S.E. \& Bojesen, A.M. (2013). Pathogenicity of wild-type and small-colony variants of Streptococcus equi subsp. zooepidemicus in layer chickens. Avian Pathology, 42, 316-322.

Sato, G. \& Miura, S. (1960). An outbreak of haemolytic streptococcal infection among chickens of a flock. II. Characteristics of the isolated streptococci. Japanese Journal of Veterinary Research, 8, 285-294.

Thayer, S. G. \& Waltman, W.D. (2013). Streptococcus and Enterococcus. In D.E. Swayne (ed.). Diseases of Poultry 13th edn (pp.977-985). Blackwell Publishing, Oxford.

Webb, K., Jolley, K.A., Mitchell, Z., Robinson, C., Newton, J.R., Maiden, M.C. \& Waller, A. (2008). Development of an unambiguous and discriminatory multilocus sequence typing scheme for the Streptococcus zooepidemicus group. Microbiology, 154, 3016-3024. 
Table 1. Bacterial analysis of organs collected from layers at necropsy.



* Hens with signs of cloacal pecking or cannibalism were excluded from bacteriological analysis

,,++++++ bacterial colonies present on the first, second and third agar streak

- : negative sample; cfu: colony forming units; NA: not analysed; S.: Streptococcus

'detection limit: $1 \times 10^{2} \mathrm{cfu} / \mathrm{g}$ 


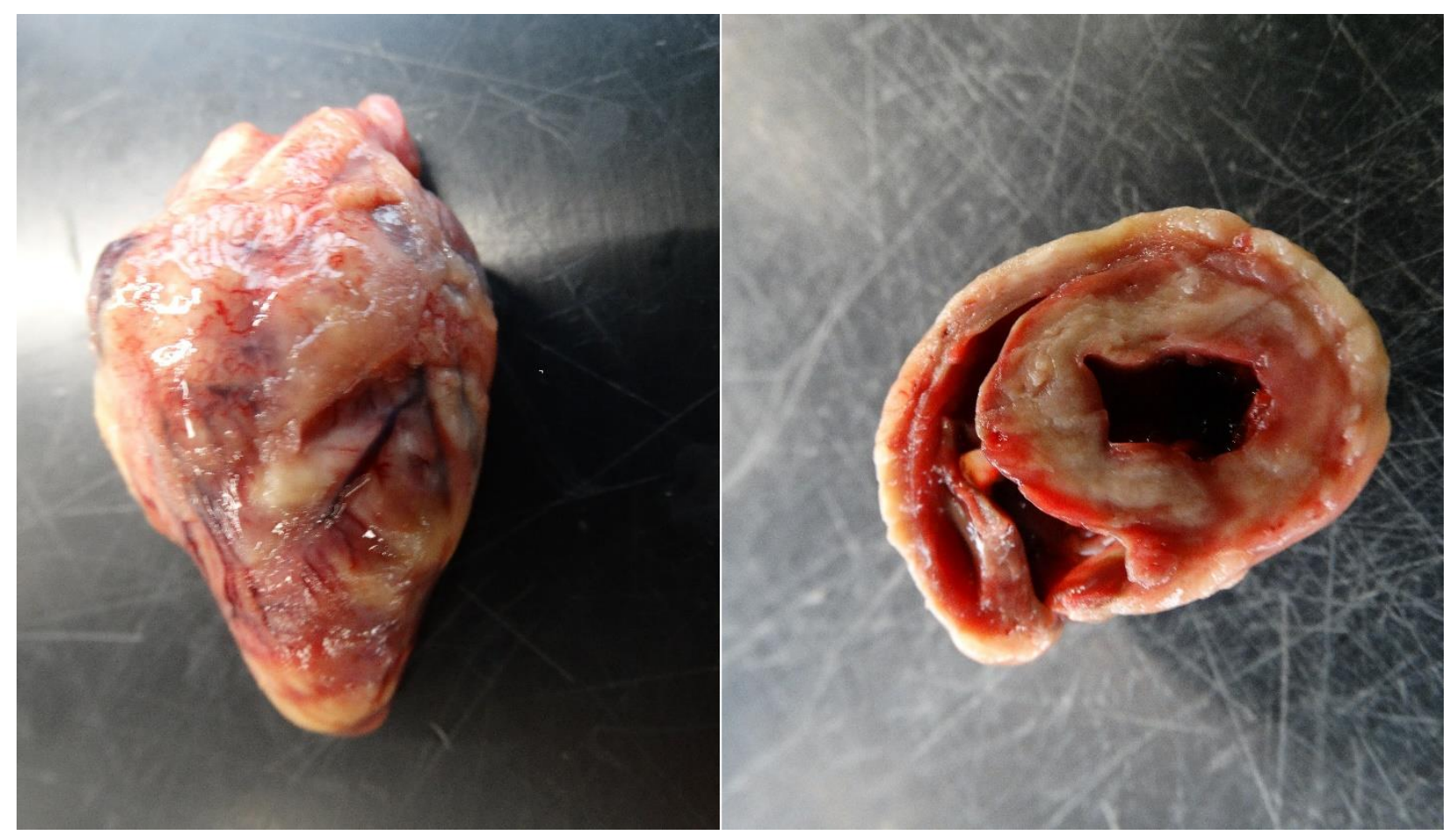

Figure 1. Heart and transverse section of the heart: Streptococcus equi subsp. zooepidemicus infection showing necrotic myocarditis.

This case is published as:

Garmyn, An, Norbert Van de Velde, Debby Braeckmans, Stef Ronsmans, Filip Boyen, Marc Verlinden. 2020. An outbreak of Streptococcus equi subsp. zooepidemicus in layers: evidence of fecal transmission. Avian Diseases, 64 (3): 343-346. 